\title{
Justesse du savoir pratique : des couleurs aux angströms dans la fabrication de circuits intégrés. Qualifications des ouvriers et des ingénieurs dans une industrie de pointe The Appropriateness of Practical Knowledge: From Colors to Angstroms in the Manufacture of Integrated Circuits. Qualifications of Workers and Engineers in a High-tech Industry
}

\section{Chantale HÉTU}

Volume 23, numéro 1, printemps 1991

Savoirs institués, savoirs informels

URI : https://id.erudit.org/iderudit/001349ar

DOI : https://doi.org/10.7202/001349ar

Aller au sommaire du numéro

Éditeur(s)

Les Presses de l'Université de Montréal

ISSN

0038-030X (imprimé)

1492-1375 (numérique)

Découvrir la revue

Citer cet article

HÉTU, C. (1991). Justesse du savoir pratique : des couleurs aux angströms dans la fabrication de circuits intégrés. Qualifications des ouvriers et des ingénieurs dans une industrie de pointe. Sociologie et sociétés, 23(1), 131-143.

https://doi.org/10.7202/001349ar

\section{Résumé de l'article}

Faire le point sur la qualification professionnelle dans une industrie de pointe conduit à se pencher sur la création des connaissances techniques, sur leur appropriation et leur diffusion dans les activités quotidiennes. Cette étude ethnographique permet: 1 ) de décrire les compétences techniques associées aux qualifications des ouvrier(e)s et des ingénieurs en comparant leurs points communs et leurs différences en réponse à la question : Quelle est la relation entre qualifications et compétences d'une part, et le partage du savoir entre groupes professionnels dans l'organisation du travail d'autre part? L'organisation du travail coordonne et délimite les connaissances techniques description de postes et savoir tacite - qu'ouvriers et ingénieurs peuvent acquérir par leurs expériences de travail. L'analyse précise ainsi les concepts de qualification et organisation proposés par E. O. Wright.
Tous droits réservés ( Les Presses de l'Université de Montréal, 1991
Ce document est protégé par la loi sur le droit d'auteur. L'utilisation des services d'Érudit (y compris la reproduction) est assujettie à sa politique d'utilisation que vous pouvez consulter en ligne.

https://apropos.erudit.org/fr/usagers/politique-dutilisation/ 


\section{Justesse du savoir pratique: des couleurs aux angströms dans la fabrication de circuits intégrés Qualifications des ouvriers et des ingénieurs dans une industrie de pointe}

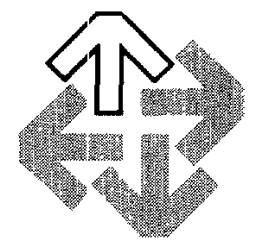

CHANTALE HÉTU

À mes yeux, les gravures sont devant moi comme une première œuvre achevée, ou plus exactement derrière moi. Puisqu'elles me paraissent déjà étranges, comme une chronique tirée de ma vie. Je dois maintenant prouver ceci à d'autres, pas à moi-même, en faisant quelque chose.

Paul Klee, Journal.

Si les réalisations techniques de l'industrie microélectronique suscitent d'emblée l'adrairation, les savoirs de leurs artisans, au cour de la production de cette technologie, paraissent difficiles à définir et à observer. Faire le point sur la qualification professionnelle dans une industrie de pointe amène à se pencher sur la création des connaissances techniques, sur leur appropriation et leur diffusion au quotidien. Dans un cadre plus général, il s'agit d'attirer l'attention sur les rapports entre diplômes et compétences en milieu de travail. La technologie se présente d'abord comme un mode de penser et d'agir des acteurs dans un contexte social particulier, ici celui du travail de production de la technologie dans une industrie de pointe en Californie.

La production de circuits intégrés et les activités de recherche et de développement dans l'industrie microélectronique soulèvent les interrogations suivantes: 1) Comment sont produites les connaissances techniques qui font partie intégrante de la qualification professionnelle? 2) Quel est le rapport entre qualifications et compétences (en tenant compte de leurs composantes) et le partage du savoir entre groupes professionnels? Il s'agit de faire ressortir comment l'organisation du travail délimite les connaissances techniques qu'ouvriers et ingénieurs peuvent acquérir et créer par leurs expériences de travail. Ayant participé aux activités d'une équipe de travail de photolithographie dans une entreprise fabriquant des composants semiconducteurs, il m'a été possible de comparer les représentations mentales que se font respectivement les ouvriers et les ingénieurs de cet objet aux fins de production. Des observations sur les lieux de travail et l'analyse des conversations au travail portant 
sur le contrôle de la qualité des produits au microscope mettent en relief certains aspects d'efficacité liés à l'efficacité et à l'idéologie touchant la qualification ${ }^{1}$.

Afin de tracer des liens entre les observations sur les pratiques de travail comme mode de penser et d'agir et le partage des connaissances techniques entre classes sociales, nous présentons les concepts de qualification et d'organisation proposés par Wright (1985). Pour saisir les connaissances techniques dans leurs manifestations concrètes, quelques notions sous-jacentes à la distribution des connaissances parmi le personnel technique appellent une définition: qualification professionnelle et, par la suite, compétence et connaissances formalisées dans la documentation technique. La comparaison des représentations mentales dont disposent les ouvrières et les ingénieurs de production pour l'inspection des circuits intégrés met en évidence quelques-unes des connaissances techniques acquises par expérience. Elles constituent un bon exemple du type de connaissances techniques que génère une industrie de pointe. Les ouvrières et les ingénieurs se fondent sur ces représentations mentales pour faire le pont entre des critères techniques objectifs et des connaissances tacites subjectives. Leurs compétences renvoient donc à une capacité d'action médiatisée par la parole et la vision; une forme de connaissance tacite.

Les qualifications ou compétences, définies par les connaissances techniques et les pratiques de travail, sont décrites tout en opérationnalisant les notions de rationalité et d'idéologie, en ce qu'elles s'insinuent au niveau le plus immédiat des modes de penser et d'agir des acteurs sociaux au travail. Il s'agit de décrire ces compétences techniques associées aux qualifications en comparant leurs points communs et leurs différences pour ensuite les expliquer en les mettant en rapport avec les points d'insertion des ouvrières et des ingénieurs dans l'organisation du travail. En conclusion, cette démarche permet d'esquisser les interrelations entre savoir pratique, science et technologie dans l'industrie, et certaines incidences idéologiques des formes de la connaissance en rapport avec les classes sociales.

\section{QUALIFICATION PROFESSIONNELLE}

Le lien entre diplômes et compétence professionnelle - sous forme de transfert de connaissances - n'est pas aussi étroit que d'aucuns l'auraient souhaité à la suite de la démocratisation de l'enseignement observée depuis trente ans (Berg, 1970; Collins, 1979). Il y a déjà longtemps, Michael Piore (1968, p. 437) faisait valoir que d'un point de vue analytique, la formation, la production et les innovations devraient être conçues comme les résultats conjoints d'un seul processus. En l'absence de changements technologiques, la formation se fait dans le processus de production, et ce pour la plus grande partie de la main-d'œuvre en Amérique du Nord. Lorsqu'il se produit un changement ou une innovation technologique, la formation s'inscrit aussi dans le processus d'innovation, d'installation et de résolution de problèmes techniques lié au nouvel équipement. En Amérique du Nord, la main-d'œuvre acquiert les habiletés nécessaires au travail au cours de brèves périodes de formation.

Quoique souvent par trop générales, les définitions de la qualification professionnelle relèvent toutes au moins deux dimensions: les compétences ou habiletés techniques objectives élaborées dans le cadre de relations de production dans une entreprise et les définitions conventionnelles du statut professionnel. Les compétences nécessaires à la production se révèlent différer des descriptions formelles des postes dans les entreprises - pour autant qu'elles existent - et de ce que prescrivent le taylorisme ou le fordisme. Habituellement, le contrôle de la production revêt deux aspects, la coordination et la discipline auxquelles

1. L'observation participante sur les qualifications dans l'industrie des semiconducteurs a été effectuée en Californie en 1985 et s'insère dans une étude plus vaste sur l'industrie microélectronique. Nous remercions pour leur soutien financier le Fonds FCAR (Québec), le Conseil de recherche en sciences humaines du Canada et la University of California. San Diego. Cet article a été en partie élaboré durant mon séjour comme chercheure invitée au département de communication de la University of California, San Diego, que je remercie de son hospitalité. 
s'ajoute une autre dimension de la qualification professionnelle: le contrôle de l'incertitude et des contingences de la production, toute activité productive comportant un degré d'incertitude et d'indétermination ${ }^{2}$.

Dans l'industrie microélectronique américaine, l'innovation technologique a tenu à des améliorations et à des changements techniques très graduels qui font désormais partie intégrante de l'innovation ou des produits mêmes. En effet, les changements techniques - miniaturisation - et l'appropriation du savoir technique fondée sur la pratique - learning by doing - ont entraîné une chute remarquable des coûts unitaires et une hausse significative de l'efficacité des procédés de fabrication (Dosi, 1984, p. 300; Braun et Macdonald, 1982; Malone, 1985). La production de la technologie est le résultat de routines organisationnelles qui sous-tendent la solution de problèmes techniques. Comme les connaissances associées à ces pratiques apparaissent à la fois spécialisées et essentiellement fugaces, on considère les activités techniques comme difficiles à décrire et, partant, les règles et procédures qui les gouvernent ne sont pas décrites en détail. La transmission de ces connaissances tacites est tributaire des contacts personnels et échappe à l'observateur extérieur (Laudan, 1984, p. 6; Polanyi, 1958, p. 49-65). Cet obstacle apparent peut être levé, comme nous le verrons.

La production de la technologie microélectronique requiert un ensemble de connaissances comprenant l'ensemble des connaissances scientifiques portant sur les propriétés fonctionnelles de l'objet technique - le circuit intégré —, les connaissances techniques sur les procédés de fabrication (chimie, etc.), et les compétences des employés. Dans cette industrie de pointe, les connaissances techniques dont le personnel est dépositaire sont à la source du processus d'innovation technologique, de sorte que des sociétés industrielles s'approprient continuellement ces connaissances comme biens productifs (Dosi, 1984, p. 288-289).

Les équipes de travail de production comprennent des ingénieurs, des superviseurs, des techniciens et des ouvriers affectés à divers postes. Dans une industrie de pointe comme la microélectronique, le personnel technique d'encadrement est plus nombreux - ingénieurs et techniciens - par rapport aux ouvriers que dans les industries conventionnelles en raison de la nature de l'évolution rapide des procédés de fabrication et des produits. Parmi les ingénieurs, environ dix pour cent sont des femmes. Elles constituent le tiers des techniciens, lesquels sont souvent des ouvriers promus en raison d'une scolarité technique et de leurs dispositions individuelles ou bien des étudiants en génie qui travaillent déjà dans l'industrie tout en étudiant. La majorité des ouvriers sont des femmes (environ soixante-dix pour cent), à qui on confie le plus souvent des tâches exigeant de la minutie. D'autres postes sont typiquement réservés aux hommes, tels la manipulation des produits chimiques. Le laboratoire crée son propre rythme. On y circule plus lentement que dans les autres aires. Il s'agit d'un monde dont le rythme est apparemment lent où les habiletés requièrent de la précision. Le travail exige une attention de tous les instants; la journée est ponctuée de décisions portant sur des détails techniques, nécessitant des jugements à propos de géométries, de mesures et de processus.

2. La littérature mentionne fréquemment ce facteur: travail et organisations (CROZIER, 1964; PERROW, 1977; STINCHCOMBE. 1959); professionnalisation (FREIDSON, 1986: SARFATTI-LARSON, 1977); science et technologie (BAXANDALL, 1986; MUKERJI, 1989; VINCENTI, 1984, 1986). Ainsi, dans l'aéronautique, la réduction de l'incertitude est un objectif en soi plutôt que la conséquence du développement des connaissances techniques en vue d'une meilleure performance des avions. La réduction de l'incertitude est à la source de l'accroissement des connaissances techniques, même à niveau fixe de performance technique. Le travail de conception architecturale ou picturale est aussi un processus comportant une série d'étapes visibles et invisibles. Ainsi, les objets sont en quelque sorte la solution à des problèmes situés dans un contexte historique. Pour une discussion de la solution de problèmes comme élément important d'une culture technique, voir BAXANDALL (1985) à propos de l'architecture, et LAUDAN (1984) et MUKERJI (1989) à propos de la science. 


\section{ORGANISATION ET QUALIFICATIONS}

Wright (1984) distingue dans la société industrielle contemporaine quatre sortes de biens productifs: les moyens de production, les qualifications, l'organisation et le processus de production. Deux de ces concepts, organisation et qualification, seront clarifiés par certains résultats de l'étude empirique que nous avons menée sur la production de circuits intégrés et les activités de recherche et de développement. Les témoignages recueillis auprès des artisans de l'industrie microélectronique confirment la pertinence d'un examen de la relation entre la distribution des qualifications et les diplômes et certificats dans la production de la technologie, c'est-à-dire les processus sociaux dans lesquels s'insèrent les connaissances et les positions ou pratiques de travail associées aux classes sociales. L'organisation - c'està-dire les conditions de la coopération coordonnée parmi les producteurs dans une division sociale du travail complexe - est une richesse productive en soi (Wright, 1984, p. 393). Plus spécifiquement, l'activité par laquelle on utilise ce bien est la prise de décision coordonnée dans le cadre d'une division technique du travail en fonction de buts. L'organisation constitue aussi un aspect important des activités de recherche et de développement technologique. Ainsi, pour favoriser une meilleure compréhension des activités liées à l'innovation technologique, on a suggéré (Mowery et Rosenberg, 1982, p. 234) de concentrer l'attention sur certaines innovations ou certains produits à travers le temps. Les composants semiconducteurs constituent à cet égard un bon exemple. Nous retiendrons donc comme unité d'analyse l'organisation au sein de laquelle s'insèrent les processus de recherche et d'innovation. Ce point de départ permet une analyse fine de l'évolution des flots d'informations menant au succès ou à l'échec dans la production d'innovations au fil du temps.

Étant donné que ce sont les rapports entre organisation du travail concrète et connaissances techniques dans le processus d'innovation qui nous intéressent au premier chef, nous allons donc tourner davantage notre attention vers la description des savoirs et des pratiques que vers les formes organisationnelles et les pratiques de gestion. Puisque l'activité par laquelle on utilise ce bien - l'organisation - est la prise de décision coordonnée dans le cadre d'une division technique du travail, Wright (1984, p. 393) affirme que certaines positions dans l'organisation ont un contrôle effectif sur une plus grande portion de ce bien que d'autres. Aussi les relations sociales de production en rapport avec ce bien prennent-elles la forme d'une autorité hiérarchique.

Leur rareté constitue la caractéristique-clé du concept de qualifications qui, succinctement, correspond selon Wright à la rareté des diplômes. certificats et licences (1984, p. 397). Comme cette assertion paraît a priori paradoxale, la relation entre qualifications ou compétences et diplômes dans une organisation donnée a besoin d'être précisée.

Il y a fondamentalement trois raisons à l'origine de la rareté relative de la qualification: tout d'abord, elle peut faire appel à des talents particuliers naturellement rares au sein d'une population; deuxièmement, la formation nécessaire à l'appropriation des habiletés peut être limitée grâce à divers mécanismes créant une rareté artificielle d'individus formés; troisièmement, un système de certification peut être établi de telle façon qu'il empêche les individus non certifiés d'être employés ou embauchés afin d'utiliser leurs qualifications ou compétences en vertu de la rareté de la qualification. (C'est nous qui traduisons.)

Grâce aux observations ethnographiques, aux interviews et aux conversations enregistrées, il est possible de décrire la distribution des compétences en relation avec l'organisation telle qu'elle ressort de la production même de la technologie. Les diplômes et certificats professionnels, ou leur absence dans le cas des ouvriers, sont, pour les individus, la clé de leur point d'insertion dans l'organisation du travail. Toutefois, les diplômes n'expliquent pas les connaissances pratiques et le savoir-faire, pas plus que les développements techniques qui ont lieu dans l'industrie.

Trois hypothèses peuvent expliquer l'incommensurabilité des connaissances techniques formelles - devis et spécifications techniques - et des pratiques de travail en ce qui 
concerne la zone d'incertitude évoquée plus tôt. Selon la première, les connaissances techniques sont subdivisées de manière déductive en directives pour chacun des postes de travail. Les ouvriers appliquent sans marge discrétionnaire ces directives que l'on présume tout à fait explicites et exhaustives. Pour la seconde, les connaissances techniques sont formalisées de façon inductive à partir des pratiques de travail des ouvriers et, une fois standardisées, elles sont redistribuées en postes individuels. La troisième hypothèse, dialectique cette fois-ci, veut que les connaissances techniques soient simultanément subdivisées en tâches assignées à des postes tout en provenant des pratiques de travail des ouvriers. Ce processus de structuration dialectique a lieu dans un contexte économique et social où les produits évoluent à un rythme rapide. Nous illustrerons cette dernière hypothèse.

Au cours de la période de formation et durant les quarts de travail, les informations sont transmises au sein de l'équipe sous forme de descriptions et de suggestions verbales pendant que chacun visualise à tour de rôle le circuit intégré au microscope. Le contenu des conversations permet de saisir les opérations effectuées au cours de la production, quoique les compétences techniques se manifestent aussi dans des pratiques nonverbales ${ }^{3}$. L'abondance des détails communiqués dans ce cadre permet de surmonter les lacunes des descriptions de postes et des devis techniques. Cette méthode d'observation de la production de routine favorise une fine analyse de la façon dont différentes occupations s'insèrent dans l'ensemble de la production.

\section{REPRÉSENTATIONS MENTALES}

Un circuit intégré se compose de fines couches de matériaux gravés sur une pastille de sillicium. Des centaines de circuits intégrés d'un demi-pouce carré sont fabriqués en superposant plusieurs couches sur une pastille de silicium, laquelle est ensuite subdivisée en autant de composants semiconducteurs. Le silicium est semiconducteur, c'est-à-dire qu'il est conducteur de courant électrique et que son degré de conductivité peut être altéré. Pour détecter les problèmes techniques et les localiser sur le circuit intégré, les ouvriers doivent apprendre à reconnaître les éléments du circuit. Aux différentes étapes de la production, visualiser un circuit intégré au microscope est analogue à la lecture d'un devis ou d'une carte topographique. On jouit d'une vue en deux dimensions d'un objet qui en possède trois.

Les représentations mentales sont une image simplifiée composée des élémentsclés à rechercher lorsqu'on observe au microscope le circuit intégré. Percevoir le premier et le deuxième points d'interconnexion sur un circuit intégré et juger de leur alignement est une des opérations les plus difficiles du processus photolithographique. Il est difficile d'abord de voir ces points minuscules et de juger de leur alignement. Les points d'interconnexion ont une importance critique puisque le courant électrique circule à travers eux d'une couche à l'autre à travers le circuit complété; sans eux, le circuit intégré ne peut fonctionner.

Quoique les postes soient standardisés conformément à ce que stipulent les techniques, la production des circuits intégrés suppose des connaissances techniques que l'on ne peut s'approprier que par la pratique. Il s'agit d'observer de quelles façons les employés transposent, grâce à leurs représentations mentales du circuit intégré, les spécifications techniques standardisées pour chaque problème ou produit particulier. Tout d'abord, la maîtrise du vocabulaire ou langage technique participe de la compréhension de cette nouvelle technologie par la pratique, de cette «maîtrise du concret» (Scribner, 1984, p. 40). À plus forte raison,

3. L'analyse sociolinguistique des conversations de travail emprunte à trois grandes tendances: la philosophie du langage (AUSTIN, 1962, SEARLE, 1969, 1975); l'analyse des conversations (GARFINKEL et SACKS, 1970) et l'analyse du discours (GUMPERZ, 1982) qui met l'accent sur les données ethnographiques. La plupart des méthodes ou modèles en analyse sociolinguistique considèrent le corpus des conversations comme une entité. En revanche, reconstituer le contexte général pertinent est crucial pour l'analyse; une attention soutenue à l'enchaînement des conduites et à l'élaboration des discours conduit à cerner ce contexte. Dans mes travaux, toutefois, l'analyse des conversations de travail selon cette dernière approche constitue un élément s'inscrivant dans une problématique plus large qui recoure aussi à d'autres données. 
pour décrire les compétences techniques à ce niveau de miniaturisation, la participation aux activités de production de même que l'enregistrement des conversations sont les conditions mêmes de l'analyse du processus de transmission des connaissances de base et des procédés techniques dans l'organisation. Ceci permet de surmonter les difficultés relatives au caractère tacite des savoirs spécialisés et d'en saisir la richesse.

L'équipe de production effectue un contrôle de la qualité de l'impression photolithographique avant de décider d'accepter les circuits intégrés tels quels ou de reprendre les dernières opérations, conformément aux critères de qualité et de quantité établis dans les devis techniques. La compétence associée à la qualification, dans le cas qui nous occupe, tient aux connaissances techniques énoncées verbalement et au jugement éclairé et exercé des ouvriers et des ingénieurs en usant des représentations mentales. Ces connaissances permettent de faire le pont entre, d'une part, l'apparente stabilité des critères techniques objectifs et des standards de qualité établis et, d'autre part, les particularités et l'incertitude de chacun des cas examinés. Les conversations de travail mettent en relief quelques éléments cognitifs des représentations mentales utilisées. Toutefois, les représentations mentales ainsi articulées sont rarement aussi explicites que durant la période d'apprentissage ou de formation; elles sont devenues implicites et tenues pour acquis de tous. Lorsque les ouvriers reconnaissent sur la pastille les critères consignés dans la documentation technique, les représentations mentales lient leurs perceptions structurantes aux traits ou caractéristiques des circuits intégrés soumis à l'inspection.

Les pratiques de travail sont avant tout des modes de penser et d'agir auxquels recourent les employés, de façon routinière ou ingénieuse, dans l'accomplissement des tâches de production. On conçoit donc le travail comme une activité sociale organisée en fonction de buts, activité qui implique une technologie et un corpus de connaissances communes (Scribner et Cole, 1981, p. 236). Cette analyse empirique du savoir entend tenir compte à la fois des aspects techniques et hiérarchiques des lieux de travail aussi bien que des processus cognitifs lorsque les employés utilisent le savoir dans des situations ou des cas particuliers pour résoudre des problèmes techniques. Pour délimiter précisément le partage des connaissances techniques, il s'agit d'établir les points communs et les différences entre les compétences des ouvriers et des ingénieurs en se fondant sur leurs conversations de travail et, en outre de se livrer à une observation ethnographique de la production. Leurs interactions et les échanges dans les conversations correspondent aux relations de production existant dans l'entreprise et, à un niveau plus général, dans l'industrie. L'observation ethnographique porte sur les tâches, la technologie et les relations sur les lieux de travail.

L'ingénieur de production et les ouvrières acquièrent des représentations mentales semblables mais distinctes du même objet technique. L'élément critique tient à ce que les ouvrières jouissent d'une vue en plongée, topologique, des circuits intégrés sur la pastille de silicium. Pour sa part, lorsque l'ingénieur examine les circuits intégrés au microscope, il les visualise selon une représentation en coupe transversale, comme dans une microphotographie. Ces facteurs influencent la perception qu'ils s'en font et rendent les ingénieurs plus conscients du rôle de l'épaisseur des couches et de leurs points d'interconnexion pour la circulation du courant électrique. Pour mieux étayer leur description verbale, l'ingénieur et les ouvrières dessinent sur le papier ou sur leurs gants de caoutchouc ce qu'ils voient à travers le microscope à mesure qu'ils le décrivent à l'intention de quelqu'un. Les descriptions verbales et les croquis montrent l'existence de deux représentations mentales distinctes.

Un jour, alors qu'il assurait la coordination technique d'une équipe, l'ingénieur fournit une longue explication sur l'alignement des points d'interconnexion. La gravure de ces points constitue une étape importante de la fabrication des circuits intégrés, et il importe de déterminer lequel a été mal aligné. Au lieu des deux points d'interconnexion habituels, quelquefois un troisième point d'interconnexion est nécessaire, comme l'expliquait l'ingénieur tout en dessinant une coupe transversale du circuit intégré. La majeure partie des explications de l'ingénieur sur la nécessité d'un troisième point d'interconnexion sont issues de sa connaissance des opérations de gravure. Il souligne que la nécessité d'un troisième point de contact 
tient, dans ce cas particulier, à un problème de fabrication non encore identifié : «Nous ne savons pas exactement pour l'instant.» «Nous effectuons des tests pour ce composant en particulier.» «Nous avons plus d'oxyde à dégager par la gravure dans les points de contact qu'il ne doit y en avoir.» Ceci constitue un exemple d'indétermination ou d'ambiguïté d'interprétation lorsqu'on emploie la représentation mentale dans des cas spécifiques.

En donnant son explication sur le troisième point d'interconnexion, l'ingénieur suppose que les ouvrières connaissent déjà les opérations de gravure acide effectuées dans le département voisin. Il décrit les opérations de gravure et leurs conséquences habituelles, faisant ressortir les écarts entre les résultats attendus et ceux des opérations observées. Ainsi, il décrit: «Théoriquement, maintenant, il va arriver ceci. Nous allons graver deux points et cela ressemblera à ceci. Voici ce que cela est censé faire.» Ensuite il marque l'écart entre «Ça grave en profondeur comme ça.» «Et on doit graver le reste ici.» Une ouvrière réalise soudainement que le deuxième point de contact est censé être gravé plus profondément que le premier point d'interconnexion. L'ingénieur de production confirme immédiatement: «Ah, oui. Mais c'est l'objectif même.» Les croquis de l'ingénieur, étayant la description verbale au fil du dialogue mettent en évidence la façon dont s'emmêlent les connaissances techniques descriptives et les connaissances tacites ou procédurales dans la production. Les informations descriptives renvoient aux connaissances techniques de base du circuit et au contenu de la documentation visant la standardisation des produits et des opérations. Le croquis ne sert pas seulement à représenter l'objet. Ce n'est pas tant le croquis une fois terminé qui est utile à la description que son caractère graduel, séquentiel, qui facilite la communication de la connaissance procédurale. Ainsi, la construction sociale des faits techniques dits objectifs est le résultat d'un processus qui relève de l'interaction sociale et de la convention dans le cadre d'une activité socialement organisée.

Finalement, l'ingénieur récapitule la description tout en faisant un croquis. Distinguant d'abord les couches ou films sur la surface de la pastille de silicium, l'ingénieur résume: «Le premier point vient jusqu'ici à peu près, et ensuite le deuxième point de contact. Voici ce qui reste.» Après un moment, l'ouvrière se rend compte à haute voix que, après les opérations de gravure, les points d'interconnexion ont servi à perforer des cylindres. Dans ce dialogue à propos d'un problème technique, on aura donc clarifié le fait que le troisième point d'interconnexion sert à atteindre le silicium lorsque la gravure du deuxième point n'a pas réussi.

On assiste à une appropriation de la connaissance dans des conversations de travail comme celle-ci, particulièrement longue et riche. Néanmoins, la spécialisation des tâches parmi les ouvriers impose des contraintes à cette appropriation-diffusion de la connaissance qui repose sur les interactions de travail. La conversation dont sont tirés les extraits cités ici rendait explicites et accessibles aux ouvrières une quantité considérable de renseignements techniques, ce que ne permettent pas les conversations habituelles. L'appropriation continue de la connaissance peut prendre des formes variées. Ainsi. un ouvrier collectionnait des pastilles de silicium pour les scruter au microscope bien que son poste ne requière pas l'utilisation du microscope. Les ouvriers mémorisent aussi les feuilles de route donnant les opérations du processus de production. Tirant parti des ressources disponibles, ils explorent et découvrent leur environnement de travail. Quelquefois, leur apprentissage se voit limité par des contraintes et, bien que motivés au départ, la frustration s'installe en raison de leur faible mobilité d'un poste à l'autre et du caractère répétitif des tâches qui leur sont assignées.

\section{ACTION MÉDIATISÉE PAR LA PAROLE ET LA VUE}

La dimension tacite de la qualification émerge lorsque les ouvriers transposent les critères objectifs et standards techniques en les interprétant d'un cas à un autre. Les ouvriers n'agissent pas sur l'objet technique de manière directement observable. À ce niveau de miniaturisation, on aurait plutôt l'impression que la capacité d'action de l'homme sur la matérialité des objets techniques est mise entre parenthèses. Toutefois, leurs actions se manifestent 
dans des actes de paroles conjointement avec leur perception visuelle de l'objet. L'exercice de la compétence des ouvriers nécessite en premier lieu une «lecture» de la matérialité la surface de la pastille de silicium. Les ingénieurs et les ouvriers utilisent fréquemment, en vue d'arriver à une décision sur un problème technique, des expressions comme: «Je dois le regarder», «J'ai vu que c'était à un niveau antérieur. « «Ça va avoir l'air de.... Leur jugement éclairé prend la forme d'une lecture préalable aux opérations à effectuer ou sert de catalyseur, particulièrement face à l'incertitude et aux contingences. Les résultats ne sont pas facilement prévisibles puisque le processus de fabrication comporte un très grand nombre d'opérations et que toutes sortes de variables peuvent jouer à toutes les étapes. Par conséquent, il vaut mieux visualiser les opérations et demeurer à l'affût des anomalies que de prévoir les résultats.

Toute remarque sur le circuit intégré est à tout le moins implicitement médiatisée par une visualisation de l'objet technique et destinée à être partagée au cours du travail. Ainsi, au début d'un long dialogue, l'ingénieur avance: «Cela me semble être un troisième point de contact» et il confirme: «C'est un troisième point de contact» visant une interprétation commune avec l'ouvrière. Graduellement le repérage des indices visuels - lignes fines ou grasses, claires ou foncées, couleurs des grilles juxtaposées, etc. - permet d'écarter l'hypothèse d'un premier ou deuxième point de contact et d'affirmer qu'il s'agit d'un troisième point d'interconnexion. L'ingénieur examine ensuite les résultats des opérations précédentes pour décider de ce qui va suivre. Insistant sur les verbes «regarder» et «voir», il communique le sens de l'action sur l'objet technique: «Si tu regardes ce que nous avons gravé ici», tout en esquissant une coupe transversale du circuit intégré. Dans un autre cas semblable, l'ingénieur dira: "Ce que nous faisons en fait - ceci n'est pas un dessin à l'échelle - mais si tu devais le regarder» en guise d'introduction à une longue description de la gravure des points d'interconnexion. À un autre moment, une ouvrière dira à l'ingénieur: «Bon, je vais faire un autre test sur cette pastille de silicium et ça aura probablement l'air beau.»

L'ingénieur commente: «Ça grave en profondeur comme ça», tout en dessinant le résultat attendu, qu'il vient tout juste de décrire: «Nous allons graver en profondeur et ça ressemblera un peu à... Voici ce que c'est censé faire.» L'emploi de pronoms de la première et troisième personnes en alternance avec le verbe «graver» marque la différence entre la capacité d'action, le sens de l'action accomplie et les variables qui font toute la difficulté du travail. L'ingénieur et les ouvrières examinent les pastilles de silicium et «voient», pour évaluer l'état de l'objet, le circuit intégré; ils identifient et nomment les caractéristiques des circuits intégrés examinés pour ensuite agir sur eux. Dans leurs actes de parole, ils accomplissent différents types d'action. Premièrement, ils diagnostiquent : «il y a de la distorsion sur cette pastille», c'est-à-dire un problème d'alignement des niveaux. Deuxièmement, ils effectuent des opérations disant: par exemple, «Nous avons gravé ces points d'interconnexion.» Troisièmement, ils décident des opérations à effectuer, tel que: «On a besoin de reprendre les opérations sur ces pastilles.» En résumé, les jugements médiatisés par une visualisation des circuits intégrés sont la condition même pour agir sur l'objet technique et mettent en valeur la compétence de l'ouvrier.

L'acquisition des représentations mentales et leur utilisation comportent des dimensions technique, sociale, et cognitive en rapport avec le contenu du poste. La construction sociale des faits techniques dits objectifs est le résultat d'un processus cognitif qui relève de l'interaction sociale et de la convention dans le cadre d'une activité socialement organisée. En outre, les faits techniques sont sociaux en ce qu'ils comportent une part d'incertitude que doivent cerner par leurs jugements ouvriers et ingénieurs en raison de l'indétermination des faits techniques et de l'indétermination du processus de fabrication. Finalement, comme la comparaison des représentations mentales le révèle, les relations sociales de production inhérentes à l'organisation du travail établissent les bornes de ce que quelqu'un peut apprendre dans la pratique à partir de sa position. L'organisation coordonne, en effet, les processus d'acquisition individuels et collectifs qui sous-tendent création, appropriation et diffusion 
des connaissances techniques, si bien que les dimensions cognitive, sociale, et technique de la technologie s'enchevêtrent dans le contenu du travail.

\section{JUSTESSE DU SAVOIR PRATIQUE: COULEURS ET ANGSTRÖMS}

Les ingénieurs se fient surtout aux indices visuels et à une connaissance plus générale des opérations impliquées dans le processus pour déterminer la situation - c'est-à-dire l'état de la pastille - et prendre des décisions. L'ingénieur de production transforme ainsi des indices visuels comme la couleur en mesure quantitative pour calibrer en angströms la profondeur, telle une description scientifique. Cependant, étant donné la subdivision des opérations et la mobilité des ingénieurs, les ouvriers n'ont pas la même familiarité avec certains indices visuels que révèle la pratique de l'inspection à la photolithographie puisqu'ils ne deviennent visibles qu'après les opérations de gravure.

À l'ouvrière qui lui demande comment se rendre compte que des points d'interconnexion ne sont pas gravés jusqu'au silicium, l'ingénieur répond essentiellement qu'il peut le détecter visuellement, à la couleur. "Alors tu peux tout simplement déduire que tu as un troisième point de contact.» Prenant pour exemple ce composant semiconducteur, l'ingénieur illustre la gamme des couleurs possibles. Depuis le beige, autour de 500 angströms, jusqu'à une couleur violette, ce qui représente de 700 à 750 angströms. «À moins de 400 angströms, ç'a l'air blanc.» Il y a donc deux sortes de mesures relatives pour juger de la profondeur des points gravés. Les couleurs servent d'indicateurs que l'ingénieur traduit quantitativement en angströms. Pour prendre une décision, les indices visuels sont plus immédiatement utiles au savoir faire de l'ingénieur qu'une description formelle en angströms. La priorité immédiate pour l'ingénieur consiste, à obtenir des indices objectifs susceptibles de justifier les prochaines étapes et d'en estimer les effets. Les couleurs signalent un état particulier du produit mais elles n'entrent pas dans l'explication scientifique de cet état ou du phénomène chimique sous-jacent.

Plus important encore, la conversation que nous avons citée montre comment les contraintes organisationnelles influencent le développement des connaissances techniques pratiques pour différentes catégories d'ouvriers dans l'organisation du travail. À l'ouvrière de photolithographie qui lui demande si les ouvrières doivent montrer à l'ingénieur les pastilles de silicium lorsqu'elles notent ce problème, l'ingénieur répond: «Tu ne le verras jamais, cependant. Ceux qui peuvent détecter ce problème sont les ouvriers à la gravure.» L'ingénieur se reporte aux ouvriers de la gravure ajoutant: «Tu vois, ce n'est pas un problème de contrôle de qualité à la photolithographie, c'est un problème de gravure. Lorsqu'ils font un contrôle à la gravure, c'est là qu'ils le voient.»

Ouvriers et ingénieurs utilisent continuellement en dialoguant les verbes «regarder» et «voir» tout en fondant leur décision sur chaque cas particulier. La cohérence par rapport à l'objet particulier et la justesse de l'action sont à la base des décisions initiales: ces décisions doivent aussi être cohérentes par rapport à un ensemble de règles et de critères techniques objectifs en ce qui a trait à la standardisation des produits et procédures. La rationalité locale, c'est-à-dire la justesse de la décision, a pour base une description détaillée du cas considéré. D'autre part, la cohérence globale repose sur une ou plusieurs caractéristiques variant systématiquement dans un échantillon de cas conçu à des fins de standardisation et de généralisation. Les statistiques de production sont ainsi à la croisée de l'universel et du singulier, de la règle et de l'exception.

Les tâches respectives des ingénieurs et des ouvriers visent à contrôler les contingences et à surmonter la part d'incertitude inhérente aux procédés manufacturiers. Au cours d'une réunion de production hebdomadaire, un ingénieur de production s'adressant aux ouvriers de cette section montrait bien que l'objectif est de fabriquer des produits de qualité à peu de frais. "Quelquefois vous demandez: "Pourquoi un ingénieur donne-t-il des réponses différentes d'une semaine à l'autre pour des cas apparemment semblables?» L'ingénieur expliqua alors que des circonstances externes et des facteurs contingents peuvent influencer 
les décisions touchant les problèmes techniques. Par exemple, si les stocks d'un produit sont bas et que les commandes sont urgentes, les ingénieurs peuvent choisir, dans les circonstances et au meilleur de leur connaissance, de poursuivre les opérations plutôt que de les reprendre. Le contexte général influence alors une décision a priori strictement technique. En outre, deux lots de composants apparemment identiques peuvent n'être pas également efficaces en termes de production, et ce pour des raisons inconnues de l'ingénieur.

Au cour de la production, les connaissances techniques pratiques consistent à examiner le circuit intégré en fonction des étapes suivantes plutôt que de rechercher une description formelle ou une explication théorique de son fonctionnement. Avant toutes choses, il convient de se demander: «Que faire?» La perception des couleurs lors de la gravure des points d'interconnexion et l'usage de locutions telles que «vider les points de contact» témoignent d'une connaissance de la production bien différente de celles que supposent les théories en chimie et en physique des solides. L'ingénieur de production donne encore quelques exemples de sa connaissance de la correspondance entre gamme des couleurs et échelle des angströms dans la conversation. Cette traduction des signes visuels en mesure quantitative est un exemple des compétences des employés (Scribner, 1984; Baxandall, 1985). Dans un contexte social défini par des relations d'autorité, comme celles qui lient l'ingénieur et les ouvriers écoutant cette explication sur la pastille, donner la mesure en angströms, n'obéit pas qu'à des impératifs d'efficacité. Elle témoigne d'une expertise et sert aussi des fins idéologiques.

\section{CONCLUSION}

La comparaison des représentations mentales des ingénieurs et des ouvrières met en évidence une appropriation différentielle des compétences techniques due à leur position respective dans l'organisation du travail. Elle éclaire les zones de connaissances techniques communes et distinctes de leurs qualifications. D'une part, les ouvriers de photolithographie et les ouvriers affectés aux postes de gravure possèdent des connaissances techniques quasi exclusives. D'autre part, les ouvriers de photolithographie et l'ingénieur de production acquièrent dans la pratique des compétences qui mobilisent des connaissances techniques tantôt communes, tantôt distinctes. À la description de leurs représentations mentales respectives s'ajoute une explication lorsqu'on met en rapport les traits communs et les différences notées avec leurs points d'insertion dans l'organisation, de même que la mobilité et la vision d'ensemble variables qui leur sont assorties.

À la position hiérarchique de l'ingénieur de production correspond une portion de la prise de décision dans l'organisation - visant la coordination - plus grande que celle des ouvriers. Les jugements des ouvriers sont néanmoins critiques, puisque l'ingénieur les sollicite et doit en tenir compte dans sa prise de décision. En effet, les ouvriers sont les tout premiers à l'affût des problèmes de production alors que le processus de fabrication est en cours. Les connaissances techniques tacites ou procédurales décrites sont le plus souvent tenues pour acquises: «c'est ce qui va sans dire». Conformément à ce que pose la troisième hypothèse que nous avons présentée, l'usage des représentations mentales pallie l'incommensurabilité des spécifications techniques et des aléas, petits et grands, de la production. La rationalité du travail des ouvriers tient à leurs connaissances et, plus encore, à l'usage qu'ils font de leurs connaissances techniques dans la pratique. La rationalité n'est pas universelle en ce sens qu'elle prend, dans une grande mesure, une forme culturelle et suppose la communication. À cet égard, l'organisation est le lieu où s'exerce une coordination des processus d'apprentissage et, par là, d'appropriation et de diffusion des connaissances techniques créées. La métaphore du rythme peut contribuer à rendre compte de la rationalité: aux fins de prévision des résultats du processus, la rationalité adopte «un rythme régulier si tout va sans dire» et «un rythme syncopé lorsque les contingences et l'ambiguité émergent» d'un processus de fabrication non encore maîtrisé (Détienne et Vernant, 1974). 
L'efficacité de cette rationalité n'a donc pas tout à fait le même sens que productivité bien qu'elle y contribue.

Le savoir pratique et les connaissances formalisées dans la documentation technique constituent deux sources de justification pour le cours d'une décision. Les compétences des ouvriers se manifestent de deux manières: dans l'appropriation individuelle du contenu de la documentation technique et, ensuite, dans la mise à jour de cette documentation. Si les ingénieurs ont la prérogative de rédiger et d'approuver les spécifications techniques, leur mise au point dépend pourtant souvent des initiatives des ouvriers ou des suggestions qu'ils émettent à la demande expresse des ingénieurs. La traduction écrite, figée en propositions, de ces compétences rend invisibles les traces des processus d'interprétation et du jugernent des ouvriers, donnant ainsi un caractère ad hoc aux connaissances techniques. Parce que ce processus est devenu invisible, la documentation tend à légitimer l'expertise de l'ingénieur et son poids idéologique dans l'organisation du travail.

Les compétences de l'ingénieur de production tiennent davantage à sa mobilité et à sa connaissance de la fabrication qu'à sa scolarité, contrairement aux hypothèses habituelles sur la place de la connaissance scientifique (Whalley, 1986, à paraître; Zussmann, 1985). Quelquefois les ingénieurs diront qu'ils possèdent une compréhension d'autant meilleure de leur travail qu'ils ont commencé à travailler comme techniciens. Au moment de quitter l'université, diplôme en poche, les ingénieurs connaissent peu ou pas du tout la fabrication des circuits intégrés. Ils doivent se familiariser avec celle-ci, en faire l'apprentissage en quelque sorte, en travaillant et en lisant des publications sur la technologie des semiconducteurs. C'est ainsi que le diplôme leur donne accès à une connaissance pratique des procédés de fabrication qui prend toute sa valeur sur le marché du travail et sur les lieux de production. Ces observations ont permis d'esquisser la façon dont s'emmêlent les dimensions cognitive, sociale et technique des qualifications dans le travail, depuis la division du travail entre groupes professionnels jusqu'au niveau des décisions de routine. Ces résultats éclairent les irterrelations entre les qualifications - au sens de compétences - , la documentation techrique, l'organisation du travail et les diplômes dans cette industrie de pointe.

Chantale Hétu Département de communication Université de Montréal C.P. 6128 , Succ. «A» Montréal (Québec) Canada $\mathrm{H} 3 \mathrm{C} 3 \mathrm{~J} 7$

\section{RÉFÉRENCES}

AUSTIN, J. L. (1962), How to Do Things with Words, Londres, Oxford University Press.

BAXANDALL, Michael (1985), Patterns of Intention. On the Historical Explanation of Pictures, New Haven et Londres, Yale University Press.

BERG, Ivar (1970), Education and Jobs; The Great Training Robbery. New York, Praeger Publishers.

BERT'AUX, Daniel, Isabelle BERTAUX-WIAME (1980), Transformation et permanence de l'artisanat boulanger en France. Une enquête sur la boulangerie artisanale par l'approche biographique. Centre d'étude des mouvements sociaux, Paris, Cordes.

BRAUN, Ernest, Stuart MACDONALD (1982), Revolution in Miniature: The History and Impact of Semiconductor Electronics Re-explored in an Updated and Revised Second Edition, Cambridge, Cambridge University Press.

CERTEAU, Michel de (1980), L'Invention du quotidien. Paris, Union générale des éditions.

CICOUREL, Aaron (1981), «Notes on the Integration of Micro and Macro-Levels of Analysis, Advances in Social Theory and Methodology Toward an Integration of Micro and Macro Sociologies. (sous la dir. de K. Knorr-Cetina et A. Cicourel), Londres, Routledge \& Kegan Paul Ltd, p. 51-80.

COLL_INS, Randall (1979), The Credential Society. An Historical Sociology of Education and Stratification, New York, Academic Press.

CROZZIER, Michel (1963), Le Phénomène bureaucratique, Paris, Seuil. 
DAGOGNET, François (1984), Philosophie de l'image, Paris, Vrin.

DELBOS, Geneviève, Paul JORION (1984), La Transmission des savoirs, Paris, Éditions de la Maison des Sciences de l'Homme.

DÉTIENNE, Marcel, Jean-Pierre VERNANT (1974), «Le cercle et le lien», dans Les Ruses de l'intelligence. La Métis chez les Grecs. Paris, Flammarion. p. 261-306.

DOSI, Giovanni (1984), Technical Change and Industrial Transformation. The Theory and an Application to the Semiconductor Industry, Londres, The Macmillan Press.

FERGUSON, Charles (1983). «The Microelectronics in Distress», Technology Review, August-September, p. 24-37.

FORM, William (1987), «On the Degradation of Skills». dans Annual Review of Sociology, vol. 13 (sous la direction de W. Richard Scott et James Short), Palo Alto. Annual Reviews Inc., p. 29-47.

FREIDSON, Eliot (1986), Professional Powers. A Study of the Institutionalization of Formal Knowledge, Chicago, Chicago University Press.

GARFINKEL, H., H. SACKS (1970), On Formal Structures of Practical Actions, dans: Theoretical Sociology (sous la direction de John McKinney et Edward Tiryakian), New York, Appleton Century Crofts, p. 338-366.

GIDDENS, Anthony (1987), Social Theory and Modern Sociology, Stanford, Stanford University Press.

GUMPERZ, John J. (1982), Discourse Strategies, Cambridge. Cambridge University Press.

HABERMAS. Jürgen (1973), La Technique et la science comme idéologie, Paris, Denoël.

KIDDER, Tracy (1981), The Soul of a New Machine, New York, Avon Books.

LAUDAN, Rachel (1984), The Nature of Technological Knowledge. Are Models of Scientific Change Relevant?, Dordrecht et Londres, D. Reidel Publishing Co.

LAVE, Jean (1988), Cognition in Practice, Cambridge, Cambridge University Press.

LAVE, Jean (1984), Tailored Learning: Education and Cognitive Skills among Tribal Craftsmen in West Africa, manuscrit inédit, School of Social Sciences, University of California, Irvine.

MALONE, Michael (1985), The Big Score. The Billion-Dollar Story of Silicon Valley, Garden City, Doubleday and $\mathrm{Co}$.

MOWERY, David, Nathan ROSENBERG (1982), «The Influence of Market Demand upon Innovation: A Critical Review of Empirical Studies», Inside the Black Box. Technologv and Economics, N. Rosenberg. Cambridge University Press, p. 193-241.

MUKERJI, Chandra (1989), A Fragile Power. Scientists and the State, Princeton, Princeton University Press.

MUKERJI, Chandra (1983). From Graven Images. Patterns of Modern Materialism, New York, Columbia University Press.

NAVILLE, Pierre (1956), Essai sur la qualification du travail, Paris, Librairie Marcel Rivière et Co.

NORMAN, Donald (1983), «Some Observations on Mental Models, » in: Mental Models, (sous la direction. de D. Gentner et A. Stevens), Hillsdale, NJ, Lawrence Erlbaum, p. 7-15.

ODDONE, Ivar, Alexandra RE, Gianni BRIANTE (1981), Redécouvrir l'expérience ouvrière. Vers une nouvelle psychologie du travail, traduction de Ivano et Marie-Laure Barsotti, Paris, Éditions sociales.

PERROW, Charles (1967), «A Framework for the Comparative Analysis of Organization», American Sociological Review, vol. 32 (avril), p. 194-204.

PIORE, Michael (1968), «On-the-job Training and Adjustment to Technological Change», Journal of Human Resources, vol. 3 (Fall), p. 335-339.

POLANYI, Michael (1962), Personal Knowledge: Towards a Post-Critical Philosophy, Chicago. University of Chicago Press.

ROSENBERG, Nathan (1972), Technology and American Economic Growth, New York, Harper \& Row Publishers.

SARFATTI-LARSON, Magali (1977), The Rise of Professionalism. A Sociological Analysis, Berkeley, University of California Press.

SCRIBNER, Sylvia (1986), «Thinking in Action: Some Characteristics of Practical Thought» in Practical Intelligence. Nature of Origins of Competence in Evervday World (sous la direction de R. Sternberg et R. K. Wagner), Cambridge, Cambridge University Press, p. 13-30.

SCRIBNER, Sylvia (1984), Special Issue on Cognitive Studies of Work. Quarterly Newsletter of the Laboratory for Comparative Human Cognition, University of California, San Diego, vol. 6, $\mathrm{n}^{\circ} 2$.

SCRIBNER, Sylvia, M. COLE (1981), The Psychology of Literacy, Cambridge, Cambridge University Press.

SEARLE, J. R. (1969), Speech Acts, Cambridge, Cambridge University Press.

SEARLE, J. R. (1975), «Indirect Speech Acts» in Peter Cole et Jerry J. Morgan (dir.), Speech Acts. Syntax and Semantics, vol. 3. New York, Academic Press.

STINCHCOMBE, A. (1959), «Bureaucratic and Craft Administration of Production: A Comparative Study», Administrative Science Quarterly, vol. 14, n² 2 (September), p. 168-187.

WHALLEY, Peter (1986), The Social Production of Technical Work, Albany, N.Y. The State University of New York Press.

WHALLEY, Peter (à paraitre), «Negotiating the Boundaries of Engineering: Professionals, Managers and Manual Work», Research in the Sociology of Organizations (sous la direction de Samuel Bacharach et al.), Westbury, CT/JAI Press. 
WOOD, Stephen (1982), The Degradation of Skill?, Londres, Hutchinson.

WRIGHT, E. O. (1984), «A General Framework for the Analysis of Class», Politics and Society (1985), Classes, Londres, Verso Editions.

ZUSS MAN, R. (1985), Mechanics of the Middle Class. Work and Politics among American Engineers, Berkeley, University of California Press.

\section{RÉSUMÉ}

Faire le point sur la qualification professionnelle dans une industrie de pointe conduit à se pencher sur la création des connaissances techniques, sur leur appropriation et leur diffusion dans les activités quotidiennes. Cette étude ethnographique permet: 1) de décrire les compétences techniques associées aux qualifications des ouvrier(e)s et des ingénieurs en comparant leurs points communs et leurs différences en réponse à la question: Quelle est la relation entre qualifications et compétences d'une part, et le partage du savoir entre groupes professionnels dans l'organisation du travail d'autre part? L'organisation du travail coordonne et délimite les connaissances techniques - description de postes et savoir tacite - qu'ouvriers et ingénieurs peuvent acquérir par leurs expériences de travail. L'analyse précise ainsi les concepts de qualification et organisation proposés par E. $O$. Wright.

\section{SUMMARY}

A review of occupational qualification in a high-tech industry leads to an examination of the creation of technical knowledge and of its appropriation and diffusion into everyday activities. The present ethnographic study has made possible the description of technical skills associated with qualifications of workers and engineers by comparing their points in common and their differences, in response to the question: "What is the relationship between qualifications and skills, on the one hand, and the sharing of knowledge between occupational groups in the work organization, on the other? Work organization coordinates and defines the limits of technical knowledge - job descriptions and tacit knowledge - that workers and engineers may acquire through their work experiences. This analysis thus clarifies the concepts of qualification and organization as proposed by E. O. Wright.

\section{RESUMEN}

Hacer el balance de la calificación profesional en una industria de vanguardia lleva a examinar la creación de los conocimientos técnicos, su apropiación y su difusión en las actividades cotidianas. Este estudio etnográfico permite 1) describir las competencias técnicas asociadas a las calificaciones de los obreros(as) y de los ingenieros comparando sus puntos comunes y sus diferencias, en respuesta a la pregunta: ¿Cuál es la relación entre calificaciones y competencias, y la repartición del saber entre grupos profesionales en la organización del trabajo? La organización del trabajo coordina y delimita los conocimientos técnicos - descripción de puestos y conocimiento tácito - que los obreros(as) e ingenieros pueden adquirir en sus experiencias de trabajo. El análisis precisa así los conceptos de calificación y organización propuestos por E. O. Wright. 